\title{
ANALYSIS OF PRECONDITIONING AND RELAXATION OPERATORS FOR THE DISCONTINUOUS GALERKIN METHOD APPLIED TO DIFFUSION
}

\author{
H. L. Atkins* \\ NASA Langley Research Center \\ Hampton, VA 23681-0001 \\ Chi-Wang Shu ${ }^{\dagger}$ \\ Brown University \\ Providence, RI 02912
}

\begin{abstract}
The explicit stability constraint of the discontinuous Galerkin method applied to the diffusion operator decreases dramatically as the order of the method is increased. Block Jacobi and block Gauss-Seidel preconditioner operators are examined for their effectiveness at accelerating convergence. A Fourier analysis for methods of order 2 through 6 reveals that both preconditioner operators bound the eigenvalues of the discrete spatial operator. Additionally, in one dimension, the eigenvalues are grouped into two or three regions that are invariant with order of the method. Local relaxation methods are constructed that rapidly damp high frequencies for arbitrarily large time step.
\end{abstract}

\section{Introduction}

The discontinuous Galerkin method allows compact spatial discretizations of any order to be formulated on arbitrary meshes. The compact discretizations are well suited to explicit time-marching methods for unsteady simulations, and are easily and efficiently implemented to run in a parallel environment. ${ }^{1}$ When applied to propagation and advection equations, the stability constraint decreases only moderately ${ }^{2}$ as the order of the method is increased. However, when applied to the diffusion operator, the explicit stability limit decreases dramatically as the order of the method is increased, ${ }^{3}$ and some degree of implicitness is necessary.

Implicit time marching methods ranging from backward Euler to a high-order implicit Runge-Kutta could be employed, depending on the level of temiporal accuracy required. Regardless of the approach, a globally implicit solution is required. The direct approach, a direct solver, destroys the compactness inlerent to the discontinuous Galerkin method along with the associated advantages. Thus we are motivated to focus on

\footnotetext{
* Senior member, Senior Research Scientist, C'omputational Modeling and Simulation Branch

$\nmid$ Professor, Division of Applied Mathematics

Copyright (c) 2001 by the American Institute of Aeronautics and Astronautics, Inc. No copyright is asserted in the l'nited States under Title 17. U.S. Code. The U.S. Government has a royalty-flee license to exercise all rights under the copyright claimed herein for Governmental Purposes. All ot her rights are reserved by the copyright owner.
}

iterative methods that are local to the element and preserve the compact character of the discontinuous Galerkin spatial discretization. One obvious choice is a block Jacobi relaxation scheme in which some or all of the local contributions to the spatial operator are treated implicitly.

This paper presents the Fourier analysis of block Jacobi and block (iauss-Seidel preconditioning operators for the discontinuous Galerkin method applied to the diffusion operator. Data from the analysis is used in the development of optimal relaxation methods that rapidly damp the high frequencies, and are well suited for use with multi-grid ${ }^{7}$ techniques. Numerical tests are presented that verify the analysis.

The first section describes the discontinuous Galerkin method as applied to diffusion, introduces required notation and formulates the particular implicit problem that is the focus of this paper. The second section presents a detailed Fourier analysis, in one dimension, of the preconditioning operators and the findings of that analysis. The third section formulates several optimal relaxation methods based on data from the analysis, and demonstrates them in mumerical tests. The fourth section extends the analysis to two dimensions and formulates optimal relaxation methods that are demonstrated in numerical tests.

\section{Methodology}

In this section, we describe in generai terms the application of the discontinuous Galerkin method to a model diffusion equation of the form

$$
\frac{\partial u}{\partial t}-\nabla \cdot[\mu \nabla u]=0
$$

Although there are several ways in which the discontinuous Galerkin method may be applied to a diffusion operator, ${ }^{3-5}$ this analysis will focus on an approach that requires a first order equation. To this end, equation (1) is recast as a set of first order equations:

$$
\begin{aligned}
\frac{\partial u}{\partial t}-\nabla \cdot \vec{q} & =0 \\
\vec{q}-\mu \nabla u & =0 .
\end{aligned}
$$




\section{Spatial Discretization}

The discontinuous Galerkin method is applied on a discrete element $\Omega_{1}$ obtained by partitioning the domain into arbitrarily shaped elements. The solution in each element is approximated in terms of a set of local basis functions $\left\{b_{l, i}\right\}$ as $\dot{u} \approx u_{l} \equiv \sum_{\{i\}} b_{l, i} u_{l, i}$ and $\vec{q} \approx \vec{q} \equiv \sum_{\{i\}} b_{l, i} \vec{q}_{l, i}$ in $\Omega_{l}$. The governing equations are projected onto each member of the basis set to give a set of equations in each element that govern the evolution of the set of unknown variables $\left\{u_{l, i}\right\}$.

$$
\begin{aligned}
\int_{\Omega} b_{l, i} & {\left[\frac{\partial u_{l}}{\partial t}-\nabla \cdot \vec{q}_{l}\right] d \Omega } \\
\int_{\Omega} b_{l, i}\left[\vec{q}_{l}-\nabla u_{l}\right] d \Omega & =0 .
\end{aligned}
$$

The gradient term is eliminated by integrating by parts to obtain the weak form

$$
\begin{aligned}
& \int_{\Omega}\left[b_{l, i} \frac{\partial u_{l}}{\partial t}-\nabla b_{l, i} \cdot \vec{q}_{l}\right] d \Omega+\sum_{\{k\}} \int_{\partial \Omega_{l, k}} b_{l, i} \vec{h}\left(\vec{q}_{l}, \vec{q}_{k}\right) \cdot \overrightarrow{d s}=0 \\
& \int_{\Omega}\left[b_{l, i} \vec{q}_{l}-\nabla b_{l, i} \cdot u_{l}\right] d \Omega+\sum_{\{k\}} \int_{\partial \Omega_{l, k}} b_{l, i} h\left(u_{l}, u_{k}\right) \overrightarrow{d s}=0
\end{aligned}
$$

where $h$ denotes a numerical flux, the subscript $k$ denotes the index of a neighboring element. $\partial \Omega_{l, k}$ denotes the boundary between $\Omega_{l}$ and $\Omega_{k}$, and $\overrightarrow{d s}$ is the outward boundary normal. Note that the variables $\left\{\vec{q}_{l}\right\}$ are simply intermediate values that can be computed directly and explicitly from the solution. In the case of advection and propagation, the numerical flux is usually an approximate Riemann flux. For diffusion, the flux is simply a wcighted average of $u$ or $\vec{q}$ such that

$$
\begin{aligned}
\vec{h}\left(\vec{q}_{l}, \vec{q}_{k}\right) & =\left(1-\phi_{l, k}\right) \vec{q}_{l}+\phi_{l, k} \vec{q}_{k}, \\
h\left(u_{l}, u_{k}\right) & =\phi_{l, k} u_{l}+\left(1-\phi_{l, k}\right) u_{k},
\end{aligned}
$$

and $\phi_{l, k}=1-\phi_{k, l}$. Evaluation of the integrals and fluxes of equation (4) leads to a semi-discrete evolution equation of the form

$$
\begin{aligned}
& \frac{\partial \mathbf{U}_{l}}{\partial t}=\mathbf{A} \cdot \mathbf{Q}_{l}+\sum_{\{k\}}\left[\left(1-\phi_{l, k}\right) \mathbf{B}_{k} \cdot \mathbf{Q}_{l}+\phi_{l, k} \mathbf{C}_{k} \cdot \mathbf{Q}_{k}\right] \\
& \mathbf{Q}_{l}=\mathbf{A} \mathbf{U}_{l}+\sum_{\{k\}}\left[\phi_{l, k} \mathbf{B}_{k} \mathbf{U}_{l}+\left(1-\phi_{l, k}\right) \mathbf{C}_{k} \mathbf{U}_{k}\right],
\end{aligned}
$$

where

$$
\begin{gathered}
\mathbf{U}_{l} \equiv\left[\begin{array}{c}
u_{l, 1} \\
u_{l, 2} \\
u_{l, 3} \\
\vdots
\end{array}\right], \quad \mathbf{Q}_{l} \equiv\left[\begin{array}{c}
\vec{q}_{l .1} \\
\vec{q}_{l .2} \\
\vec{q}_{l .3} \\
\vdots
\end{array}\right], \\
\mathbf{A}=\left[\vec{a}_{i, j}\right]=\mathbf{M}^{-1}\left[\int_{\Omega} \nabla b_{l, i} \cdot b_{l, j} d \Omega\right],
\end{gathered}
$$

$$
\begin{aligned}
& \mathbf{B}=\left[\vec{b}_{i, j}\right]=-\mathbf{M}^{-1}\left[\int_{\partial \Omega_{l, k}} b_{l, i} b_{l, j} \overrightarrow{d s}\right], \\
& \mathbf{C}=\left[\vec{c}_{i, j}\right]=-\mathbf{M}^{-1}\left[\int_{\partial \Omega_{l, k}} b_{l, i} b_{k, j} \overrightarrow{d s}\right],
\end{aligned}
$$

and

$$
\mathbf{M}=\left[m_{i, j}\right]=\left[\int_{\Omega} b_{l, i} b_{l, j} d \Omega\right] .
$$

To facilitate the analysis, it is assumed that the domain has been uniformly partitioned such that the matrices $\mathbf{A}, \mathbf{B}_{k}$ and $\mathbf{C}_{k}$ are the same for all elements. Further more, in this study we will examine only the so called "mixed LR" scheme $e^{3,5}$ in which $\phi_{l, k}$ is either zero or one on each boundary segment $\partial \Omega_{l, k}$, and $\phi_{l, k}$ is assigned in a regular and repeating pattern.

\section{Temporal Discretization}

In previous works dealing with propagation, ${ }^{2,6} \mathrm{ex}-$ plicit Runge-Kutta was used to advance the solution in time. However, the stability bound of such an explicit method is inversely proportional to the maxinum eigenvalur of the spatial operator. When discontimuous Galerkin is applied to the diffusion operator, the maximum eigenvalue grows in magnitude approximately proportional to $p^{4}$, and the explicit approach becomes impractical for most problems. An implicit time marching method would clearly remedy the problem; however, a direct inversion of the resulting matrix would destroy the compactness of the discontinuous Galerkin. Thus in the present work, we consider implicit evolution methods that are solved by local relaxation methods. and we are interested in the analysis of the relaxation process.

Given a general evolution equation of the form

$$
\frac{\partial u}{\partial t}=R(u)
$$

where $R(u)$ denotes a spatial operator, all implicit methods involve solving an equation of the form

$$
\begin{aligned}
\frac{u^{n}-u^{n-1}}{\Delta t}=\frac{\alpha R\left(u^{n}\right)}{(\Delta x)^{2}}+ & H\left(u^{n-1}, u^{n-2} \ldots,\right. \\
& \left.R\left(u^{n-1}\right), R\left(u^{n-2}\right), \ldots\right),
\end{aligned}
$$

where the superscript $n$ may denote either the time step $(t=n \triangle t)$ or a step within the Runge-Kutta sequence, and $\alpha$ and $H$ depend on the specifics of the method. In the present analysis, we are concernod only in the construction and stability of an iterative method for the solution $u^{n}$ in equation (6). Thus, we assume the discrete evolution equation (6) is stable in time, possesses the desired order properties, and that old values of the solution $u^{n-1}, u^{n-2}, \ldots$ are known.

Within the scope of a Fourier analysis, the terms involving the old values of the solution have no effect 
on the stability of a relaxation scheme. Thus it is sufficient to examine a much simplified form of equation (6) given by

$$
\mathcal{R}(\lambda, v) \equiv \lambda R(v)-v=0,
$$

where $\lambda=\alpha \Delta t /(\Delta x)^{2}$, and $v \equiv u^{n}$. Including $\alpha$ in the $\lambda$ term eliminates all explicit dependence on the specific evolution method, and the conclusions of the following analysis apply to all time evolution methods that can be written in the form of equation (6). Because this class of evolution methods includes firstorder backward Euler, the following analysis also has implications for steady-state solution methods as well.

\section{Fourier Analysis}

We examine the eigenvalues of the Fourier transform of equation (7), and the effects that two preconditioners have on those eigenvalues. For the spatially discrete discontinuous Galerkin method given in equation (5) but specialized to one dimension, equation (7) becomes

$$
\begin{aligned}
\mathcal{R}\left(\lambda, \mathbf{U}_{l}\right) & =\lambda\left\{\mathbf{A} \mathbf{Q}_{l}+\left[\mathbf{B}_{l+\frac{1}{2}} \mathbf{Q}_{l}+\mathbf{C}_{l-\frac{1}{2}} \mathbf{Q}_{l-l}\right]\right\} \\
& -\mathbf{U}_{l} \\
\text { and } \mathbf{Q}_{l} & =\mathbf{A} \mathbf{U}_{l}+\left[\mathbf{C}_{l+\frac{1}{2}} \mathbf{U}_{l+1}+\mathbf{B}_{l-\frac{1}{2}} \mathbf{U}_{l}\right] .
\end{aligned}
$$

The subscript $l \pm \frac{1}{2}$ is a shorthand for the clouble subscript $l, l \pm 1$. and $\phi_{l \pm \frac{1}{2}}$ is assigned as follows: $\phi_{l+\frac{1}{2}}=1$ $\phi_{l-\frac{1}{2}}=0$. Eliminating $\mathbf{Q}$ gives

$$
\begin{aligned}
\mathcal{R}\left(\lambda, \mathbf{U}_{l}\right) & =\lambda\left\{[ \mathbf { A } + \mathbf { B } _ { l + \frac { 1 } { 2 } } ] \left[\mathbf{A} \mathbf{U}_{l}\right.\right. \\
& \left.+\left(\mathbf{C}_{l+\frac{1}{2}} \mathbf{U}_{l+1}+\mathbf{B}_{l-\frac{1}{2}} \mathbf{U}_{l}\right)\right] \\
& +\mathbf{C}_{l-\frac{1}{2}}\left[\mathbf{A} \mathbf{U}_{l-1}+\right. \\
& \left.\left.+\left(\mathbf{C}_{l+\frac{1}{2}} \mathbf{U}_{l}+\mathbf{B}_{l-\frac{1}{2}} \mathbf{U}_{l-1}\right)\right]\right\}-\mathbf{U}_{l}=0 \\
& =\lambda \mathbf{C}_{l-\frac{1}{2}}\left(\mathbf{A}+\mathbf{B}_{l-\frac{1}{2}}\right) \mathbf{U}_{l-1} \\
& +\left\{\lambda \left[\left(\mathbf{A}+\mathbf{B}_{l+\frac{1}{2}}\right)\left(\mathbf{A}+\mathbf{B}_{l-\frac{1}{2}}\right)\right.\right. \\
& \left.\left.+\mathbf{C}_{l-\frac{1}{2}} \mathbf{C}_{l+\frac{1}{2}}\right]-\mathbf{I}\right\} \mathbf{U}_{l} \\
& +\lambda\left(\mathbf{A}+\mathbf{B}_{l+\frac{1}{2}}\right) \mathbf{C}_{l+\frac{1}{2}} \mathbf{U}_{l+1} \\
& \equiv \mathcal{A}(\lambda) \mathbf{U}_{l-1}+\mathcal{B}(\lambda) \mathbf{U}_{l}+\mathcal{C}(\lambda) \mathbf{U}_{l+1}
\end{aligned}
$$

The Fourier transform is obtained by substituting $\mathbf{U}_{l}=\tilde{\mathbf{U}} e^{i \theta l}$ where $i \equiv \sqrt{-1}$, and $0 \leq \theta \leq \pi$, to give

$$
\begin{aligned}
\mathcal{R}(\lambda, \overline{\mathbf{U}}) & =\left(\mathcal{A}(\lambda) e^{-\dot{i} \theta}+\mathcal{B}(\lambda)+\mathcal{C}(\lambda) \epsilon^{i \theta}\right) \tilde{\mathbf{U}} \\
& \equiv \tilde{\mathcal{R}}(\lambda, \theta) \tilde{\mathbf{U}}
\end{aligned}
$$

The preconditioned residual has the form $l^{-1} \mathcal{R}(\lambda, v)$. 'The preconditioning operator for block Jacobi is obtained from equation (9) by retaining only the terms involving $\mathbf{U}_{l}$.

$$
\begin{aligned}
\mathbf{P}_{b j} & =\boldsymbol{B}(\lambda) \mathbf{U}_{l} \\
& =\left\{\lambda \left[\left(\mathbf{A}+\mathbf{B}_{l+\frac{1}{2}}\right)\left(\mathbf{A}+\mathbf{B}_{l-\frac{1}{2}}\right)\right.\right. \\
& \left.\left.+\mathbf{C}_{l-\frac{1}{2}} \mathbf{C}_{l+\frac{1}{2}}\right]-\mathbf{I}\right\} \mathbf{U}_{l} \\
\tilde{\mathbf{P}}_{b j} & =\mathcal{B}(\lambda) .
\end{aligned}
$$

For block Gauss-Seidel, preconditioner is defined by dropping terms involving $U_{l+1}$.

$$
\begin{aligned}
\mathbf{P}_{g s} & =\boldsymbol{B}(\lambda) \mathbf{U}_{l}+\mathcal{A}(\lambda) \mathbf{U}_{l-1} \\
& =\left\{\lambda \left[\left(\mathbf{A}+\mathbf{B}_{l+\frac{1}{2}}\right)\left(\mathbf{A}+\mathbf{B}_{l-\frac{1}{2}}\right)\right.\right. \\
& \left.\left.+\mathbf{C}_{l-\frac{1}{2}} \mathbf{C}_{l+\frac{1}{2}}\right]-\mathbf{I}\right\} \mathbf{U}_{l} \\
& +\lambda \mathbf{C}_{l-\frac{1}{2}}\left(\mathbf{A}+\mathbf{B}_{l-\frac{1}{2}}\right) \mathbf{U}_{l-1} \\
\tilde{\mathbf{P}}_{g s} & =\left(\boldsymbol{B}(\lambda) e^{-i \theta}+\mathcal{A}(\lambda)\right) .
\end{aligned}
$$

\section{Analysis of Eigenvalues}

The eigenvalues of $\tilde{\mathcal{R}}$ and $\tilde{\mathbf{P}}^{-1} \dot{\mathcal{R}}$ are computed at 50 values of $\theta$ that are uniformly distributed between 0 and $\pi$, and for $\lambda$ ranging from $10^{-8}$ to $10^{8}$. If $p$ denotes the degree of the basis functions, then in one dimension there are $p+1$ eigenvalues for earh value of $\theta$. Figure 1 shows the real component of all eigenvalues of $\tilde{\mathcal{R}}$ for $p$ equals $1-5$ and with $\lambda=1.0$ (the imaginary part is zero). The solid line is proportional to $p^{4}$ and closely matches the growth in the magnitude of the eigenvalues. Figures $2(a)$ and $2(b)$ show the eigenvalues when block Jacobi and block GaussSeidel preconditioning are used, respectively. Not only are the eigenvalues bounded for all $p$ but they are grouped into two or three regions. Both preconditioners place one eigenvalue near zero, block Jacobi has one eigenvalue near -2.0 , and all of the remaining eigenvalues are mapped exactly into -1.0 . The variation in the eigenvalues within each region indicates the degree to which the eigenvalue varies with $\theta$. A rlear trend observed in both cases is that the eigenvalues become more tightly grouped as $p$ increases. CiaussSeidel is not a symmetric operator, and consequently, the imaginary component of its eigenvalues is not zero. However, the irnaginary component is small relative to the real component, so all following results will examine either the real component or the absolute value of the eigenvalues (denoted as the absolute eigenvalue).

The above results are for $\lambda=1$, but similar trends are observed for large and small $\lambda$. Figures $3(\mathrm{a})$ and 3 (b) show the eigenvalues for block Jacobi preconditioning with $\lambda=100$ and 0.01 , respectively. With $\lambda=100$ the eigenvalue pattern is nearly identical to that of $\lambda=1$. With $\lambda=0.01$ the cigenvalues are more tightly grouped with respect to $\theta$, but the maximmm and minimum eigenvalue are not as close to 0.0 and 
-2.0, respectively. Similar trends were observed for block Gauss-Seidel.

While the largest absolute eigenvalue limits the time step of an explicit method, the smallest absolute eigenvalue is the least damped and will limit the convergence of an iterative method. Clearly both preconditioners reduce the maximum absolute eigenvalue, but this is of little value if the minimum absolute eigenvalue is equally reduced. Thus, another metric for a preconditioner is the ratio of the maximum absolute eigenvalue to the minimum absolute eigenvalue. Figures $4(\mathrm{a}-\mathrm{c})$ show this ratio of absolute eigenvalues with no preconditioning, with block Jacobi preconditioning, and with block Gauss-Seidel preconditioning, respectively. (Note: with no preconditioning the minimum absolute eigenvalue is always 1.0 , so the ratio is equal to the maximum absolute eigenvalue, and the data is just the negative of that shown in figure I; however, the data is redrawn on a log scale to facilitate comparison with the other two methods.) Block Jacobi reduces the ratio by about an order of magnitude (at $p=5$ ). Block Gauss-Seidel further reduces the ratio by about half an order (again at $p=5$ ). So both preconditioners provide considerable improvement in convergence, but not the three orders of magnitude that one might infer simply by looking at the reduction of maximum eigenvalues.

\section{Optimal Relaxation Schemes}

The tight grouping of eigenvalues means entire groups of the error modes can be rapidly damped, sometimes completely damped, by the following simple update method

$$
\mathbf{U}^{k+1}=\mathbf{U}^{k}+\omega \mathbf{P}^{-1} \mathcal{R}\left(\lambda, \mathbf{U}^{k}\right)
$$

The stability of this relaxation step is given by $G(\omega, \sigma(\theta)) \equiv|1+\omega \sigma(\theta)|$ where $\sigma(\theta)$ is any eigenvalue of the operator $\tilde{\mathbf{P}}^{-1} \dot{\mathcal{R}}$. By choosing $\omega=1$, all the error modes with an eigenvalue of -1.0 are completely damped. Similarly choosing $\omega=0.5$ will rapidly damp the error mode with an eigenvalue near -2.0 . This leaves a single error mode associated with the eigenvalue near zero, which is not readily damped by any choice of $\omega$ that is not unstable for the other error modes. The block Gauss-Seidel method can be overrelaxed with $\omega$ approaching 2 for large $p$, but this provides only a slight improvement in the damping rate of the slow error mode.

However, if one considers the combined effect of a sequence of relaxation steps

$$
\mathbf{U}^{k+1}=\mathbf{U}^{k}+\omega_{k} \mathbf{P}^{-1} \mathcal{R}\left(\lambda, \mathbf{U}^{k}\right), \quad k=1 \ldots K^{-}
$$

each with a different $\omega$, then it is possible to choose a sequence of $\omega_{k}$ that will rapidly damp all eigenmodes. In the following sections, we present several criteria for selecting an optimal sequence of $\omega$ for block
Gauss-Seidel and block Jacobi for a fixed $\lambda$. We then construct a curve fit that accounts for the dependence of the wik sequence on $\lambda$.

\section{Block Gauss-Seidel}

For block Crauss-Seidel, we consider a two step sequence whose stability is given by

$$
G_{g s}\left(\omega_{2}, \sigma(\theta)\right)=G(1.0, \sigma(\theta)) G\left(\omega_{2}, \sigma(\theta)\right),
$$

and we choose $u_{2}$ to obtain an optimal damping rate. By choosing $\omega_{1}=1.0$, the first step completely damps all error modes with an eigenvalue of -1.0 and the analysis can focus the single remaining error mode whose eigenvalue is near 0.0 . This slowly decaying error mode, denoted as $\sigma_{1}(\theta)$, is dominated by its real component which varies monotonically with $\theta$. 'This behavior is typical for all $p$ and $\lambda$ examined: $p \leq 5$ and $10^{-8} \leq \lambda \leq 10^{8}$.

In the following we examine two criteria for choosing $\omega_{2}$. The first approach minimizes $G_{g}$ s across the entire spectrum of $\theta$ by requiring

$$
G_{g s}\left(\omega_{2}, \sigma_{1}(0)\right)=G_{g s}\left(\omega_{2}, \sigma_{1}(\pi)\right) .
$$

Figure 5 shows $G_{g s}$ for $\sigma_{1}(0)$ (solid) and $\sigma_{1}(\pi)$ (dashed) as a function of $\omega_{2}$ for $p=3$, and for $\lambda=0.01 .0 .1,1.0$ and 10.0 . The symbols show $G_{g^{*}}\left(\omega_{2}, \sigma_{1}(\theta)\right)$ for a uniform sampling of $\theta$ which gives an indication of the variation of $G_{y s}$ with $\theta$ at a fixed $\omega_{2}$. The symbols also verify that either $G_{g s}\left(\omega_{2}, \sigma_{1}(0)\right)$ or $G_{g s}\left(\omega_{2}, \sigma_{1}(\pi)\right)$ bounds $G_{g s}\left(\omega_{2}, \theta\right)$ above when $\lambda$ is not small, and their intersection defines an optimal $\omega_{2}$ over all $\theta$. For extremely small $\lambda$, neither $G_{g s}\left(\omega_{2}, \sigma_{1}(0)\right)$ or $G_{g s}\left(\omega_{2}, \sigma_{1}(\pi)\right)$ bounds $G_{g s} ;$ however equation $(17)$ is still a suitable criterion for minimizing $G_{g s}$ over all $\theta$. Although the the damping rate is stable for all $\lambda$. the damping rate rises quickly with $\lambda$, and is unacceptablely high at $\lambda=10.0$. Also, when $\lambda$ is large, $\left(i_{g s}\left(\omega_{2}, \sigma_{1}(\pi)\right)\right.$ grows rapidly with $\omega_{2}$ and quickly becomes unstable. The sensitivity to $\omega_{2}$ is an issue because the analysis can only be performed for the idealized case of a uniform grid. In a realistic case in which $\lambda$ varies due to non-uniformity in the mesh. the actual optimal value of $\omega_{2}$ may be different from that predicted by the analysis, and the predicted optimal value may result in an unstable method.

$A$ second and more robust approach is to minimize $G_{g s}$ in the high frequency portion of the spectrum $(\theta>$ $\pi / 2$ ) to obtain a relaxation scheme that is well suited as a multigrid ${ }^{\top}$ smoother. To this end, we require that

$$
G_{g s}\left(\omega_{2}, \sigma_{1}(\pi / 2)\right)=G_{g s}\left(\omega_{2}, \sigma_{1}(\pi)\right) .
$$

Figure 6(a) shows the $\omega_{2}$ obtained by solving equation (18) for a range of $\lambda$. Also shown is the curve defined by

$$
\omega_{f}=\frac{\omega_{m}+1+\left(\omega_{m}-1\right) \tanh \left(a * \log _{10}(\lambda)+b\right)}{2}
$$


which provides an accurate fit of $\omega_{2}$ for all $\lambda$. The roefficients $a$ and $b$ are obtained from a least square fit over the linear subrange seen in figure $6(\mathrm{~b})$, and $\omega_{m}$ is the maximum value of $\omega_{2}$, taken here to be the value of $\omega_{2}$ when $\lambda=10^{8}$. From figure 7 , which shows the dependence of $G_{g s}$ on $\omega_{2}$ at $\lambda=10^{6}$ (similar to figure 5), we can see that large variations in $\omega_{2}$ can occur before the method becomes unstable, and that small variations do not significantly degrade the damping rate. Hence, this criterion produces relaxation methods that are robust with regard to any discrepancies that might occur between the predicted optimal $\omega_{2}$ and actual values. Figures $8(\mathrm{a})$ and $8(\mathrm{~b})$ show the behavior of $\sqrt{G_{g s}\left(\omega_{f}\right)}$ as a function of $\theta$ and $\lambda$, respectively. The maximum value over the high frequency spectrum generally occurs at $\theta=\pi / 2$, and rises to $\approx 0.53$ for large $\lambda$. So one would expect this relaxation method to be an excellent smoother for a multigrid method. It should be noted that, because the first relaxation step completely damps all error modes of this idealized linear problem except for the $\sigma_{1}(\theta)$ mode, it is possible to perform a single relaxation step with $\omega=1.0$ followed by any number of relaxation steps with $\omega=\omega_{f}$. In this case the asymptotic convergence rate would reduce to $(0.53)^{2}=0.28$. In the above discussion, $p=3$ has been used to illustrate the procedure. The same analysis has been applied to all $p \leq 5$ and the results are summarized in Table 1 . We find that the asymptotic convergence rate is essentially constant as $p$ increases.

\section{Block Jacobi}

For block Jacobi, we consider a sequence of three relaxation steps in which $\omega_{1}=0.5, \omega_{2}=1.0$ and $\omega_{3}$ is chosen to provide optinal damping of the high frequency portion of the spectrum, $\theta>\pi / 2$. The stability of such a method is given by

$$
G_{b j}\left(\omega_{3}, \sigma(\theta)\right)=G(0.5, \sigma(\theta)) G(1.0, \sigma(\theta)) C_{r}\left(\omega_{3}, \sigma(\theta)\right)
$$

where $\sigma(\theta)$ is any eigenvalue of $\tilde{\mathbf{P}}_{b j}^{-1} \dot{\mathcal{R}}$. Also let $\sigma_{1}(\theta)$ and $\sigma_{2}(\theta)$ denote the maximum and minimum eigenvalues, respectively. Because the the minimum eigenvalue $\sigma_{2}(\theta)$ is near but not exactly -2.0 , the associated error mode is not completely damperl by the relaxation step with $\omega_{1}=0.5$ and this eigenmode must be considered when determining an optimal $\omega_{3}$. In particular, we choose $\omega_{3}$ by examining the intersections of the functions $G_{b j}\left(\omega_{3}, \sigma_{1}(\pi)\right), G_{b j}\left(\omega_{3}, \sigma_{2}(\pi)\right)$, $G_{b j}\left(\omega_{3}, \sigma_{1}(\pi / 2)\right)$ and $G_{b j}\left(\omega_{3}, \sigma_{2}(\pi / 2)\right)$. As shown in figure 9 for $p=3$ and $\lambda=1.0$, the optimal value of $\omega_{3}$ is associated with the maximum intersection of these curves. For the case shown in figure $9, \omega_{3} \approx 6.7$. This process is repeated for $\lambda$ ranging from $10^{-8}$ to $10^{8}$ and fitted by equation (19) as done previously for block Gauss-Seidel. Figures $10(a)$ and $10(b)$ show the behavior of

$G_{m}\left(\omega_{3}, \theta\right) \equiv\left(\max \left(G_{b j}\left(\omega_{3}, \sigma_{1}(\theta)\right),\left(i_{b j}\left(\omega_{3}, \sigma_{2}(\theta)\right)\right)\right)^{1 / 3}\right.$ with respect to changes in $\theta$ and $\omega_{3}$, respectively. The damping rate for the high-frequency portion of the spectrum does not increase above $\approx 0.76$ as $\lambda$ becomes large. Also, the damping rate increases slowly as $\omega_{3}$ varies from the optimal value indicating that the method is robust in this respect. This analysis has been performed for all $p \leq 5$ and results are summarized in Table 2. As seen previously, the asymptotic convergence rate is nearly constant with respect to $p$.

\section{Numerical Examples}

In this section we provide a few numerical examples that show that the performance predicted by the analysis are realistic and achievable. Details of the numerical implementations are not provided here but are throughly described in the references 3 and 7 . The block Jacobi preconditioned discontinuous Galerkin method is applied to the one dimensional heat equation with periodic boundary conditions

$$
\begin{aligned}
& \partial u / \partial t=\nabla^{2} u \\
& u(0, x)=\sin (\pi x) \quad-1 \leq x \leq 1
\end{aligned}
$$

in which the solution is approximated by polynomials of degree $p=5$. Tests are performed in which the solution is evolved in time using either backward Euler or third order implicit Runge-Kutta and with $\Delta t /(\Delta x)^{2}=10$. However, here we are interested only the convergence of the temporal residual at each time step, or each Runge-Kutta stage, which is similar for both evolution methods. Figure 11 shows the convergence histories of a typical stage obtained by block Jacobi relaxation with constant $\omega=1.0$ and opt $\mathrm{i}$ mal with the three-stage $\omega$ sequence. Also shown is the convergence of block Jacobi relaxation with optimal three stage $\omega$ sequence combined with a two-level multigrid method. The multigrid method is a standard $V$-cycle with 6 relaxation steps on the fine grid betwen coarse grid cycles. The coarse grid solution is converged to machine zero by block Jacobi relaxation to simulate the effect of multiple grid levels. The case using the optimal $\omega$ sequence without multigrid shows some improventent in the clamping rate over that of constant $\omega$. The number of relaxation steps required to drive the residual to $10^{-12}$ drops from 9706 to 1731; however, this improvement is not sufficient to make this approach practical. With multigrid, the convergence improves dramatically and only 276 relaxation steps are required to drive the residual to $10^{-12}$. This corresponds to a convergence rate of $10^{-12 / 275}=0.905$, and represents a 25 fold reduction in work when compared to a sixth-order explicit Runge-Kutta method which has an explicit stability constraint $^{3}$ of $\Delta t / \Delta x^{2}=0.0015$.

\section{Extension to Two Dimensions}

The following sections describe the results of the two dimensional analysis, construction of optimal relaxation schemes for block Jacobi and mumerical results. 


\section{Two Dimensional Analysis}

In two dimensions, equation ( 7 ) has the form

$$
\begin{aligned}
\mathcal{R}\left(\lambda, \mathbf{U}_{i, j}\right) & =\mathcal{A}(\lambda) \mathbf{U}_{i-1, j}+\mathcal{B}(\lambda) \mathbf{U}_{i, j}+\mathcal{C}(\lambda) \mathbf{U}_{i+1, j} \\
& +\mathcal{D}(\lambda) \mathbf{U}_{i, j+1}+\mathcal{E}(\lambda) \mathbf{U}_{i, j-1}
\end{aligned}
$$

The Fourier transform is obtained by substituting $\mathbf{U}_{i, j}=\dot{\mathbf{U}} e^{i\left(\theta_{x} i+\theta_{y} j\right)}$ to give

$$
\begin{aligned}
\mathcal{R}\left(\lambda, \overline{\mathbf{U}_{i, j}}\right) & =\mathcal{A}(\lambda) e^{-i \theta_{x}}+\mathcal{B}(\lambda)+\mathcal{C}(\lambda) e^{i \theta_{x}} \\
& +\mathcal{D}(\lambda) e^{i \theta_{y}}+\mathcal{E}(\lambda) e^{-i \theta_{y}} \\
& \equiv \mathcal{R}\left(\lambda, \theta_{x}, \theta_{y}\right) \dot{\mathbf{U}}
\end{aligned}
$$

The preconditioning operator for block Jacobi is simply $\mathbf{P}_{b j}=\mathcal{B}(\lambda) \mathbf{U}_{l}$ and $\tilde{\mathbf{P}}_{b j}=\mathcal{B}(\lambda)$.

Typical eigenvalues are shown in figures $12(a)$ 12(c). There are 21 eigenvalues for the fifth-order method $(p=4)$. Figures $12(a)$ and $12(b)$ show the range of each eigenvalue as the wave numbers vary over the complete range $0 \leq \theta_{x}, \theta_{y} \leq \pi$. Figure $12(\mathrm{c})$ shows a contour plot of the fifth eigenvalue. The analysis predicts that both block Gauss-Seidel and block Jacobi preconditioners bound the eigenvalues, as seen in the one dimensional analysis. Several eigenvalues are mapped into -1.0 , however, the remaining eigenvalues are not tightly gromped but are distributed over the range 0 to -1.0 for block Gauss-Seidel and 0 to -2.0 for block Jacobi.

\section{Optimal Relaxation}

The eigenvalues are not tightly grouped making it necessary to design a relaxation scheme that optimally damps a broad spectrum. We choose a sequence of three relaxation steps, similar to the previous approach for block Jacobi; however, all three relaxation factors are allowed to vary freely. The amplification is given by

$$
G g_{2 d}\left(\omega_{1}, \omega_{2}, \omega_{3}, \sigma\right)=G\left(\omega_{1}, \sigma\right) G\left(\omega_{2}, \sigma\right) G\left(\omega_{3}, \sigma\right)
$$

The objective of the optimization, illustrated in figure 13 , is to minimize $\left(i_{2 d}\right.$ for all $\sigma$ in the range $-2.0<$ $\sigma<\sigma^{*} . \sigma^{*}$ denotes the largest eigenvalue in the highfrequency portion of the spectrum and it varies with $\lambda$ and $p$. Note that we are not explicitly concerned with the dependence of $\sigma$ on $\theta$. This optimization is accomplished by equating

$$
G_{2 d}\left(\sigma^{*}\right)=G_{2 d}\left(\sigma_{a}\right)=G_{2 d}\left(\sigma_{b}\right)=G_{2 d}(-2.0)
$$

where $\sigma_{a}$ and $\sigma_{b}$ denote $\sigma$ at the local extrema of $G_{2 d}(\sigma)$ and are given by $\partial G_{2 d} / \partial \sigma=0.0$. Equation (24) is quadratic, which allows $\sigma_{a}$ and $\sigma_{b}$ to be determined analytically as functions of $\omega_{1}, \omega_{2}$ and $\omega_{3}$. Equation (24) can now be solved to determine $\omega_{1}, \omega_{2}$ and $\omega_{3}$. Table 3 gives $\sigma^{*}$ and the optimal $\omega$ sequence for a range of $\lambda$ and for $p=4$. $\omega_{1}$ and $\omega_{2}$ are nearly constant except at extremely small $\lambda$, and $\omega_{3}$ is accurately fitted by a tanh distribution to give

$$
\omega_{3}=3.72+2.72 \tanh \left(1.022 \log _{10}(\lambda)+1.469\right)
$$

Figure 14 shows the asymptotic convergence rate predicted by the analysis which approaches 0.93 as $\lambda$ becones large.

\section{Numerical Examples}

Figure 15 shows the convergence history from several numerical tests. The test case is the twodimensional heat equation with periodic boundary conditions and with the same initial condition as used in the one dimensional test described previously. All results are for the fifth-order $(p=4)$ discontinuous Galerkin method with block Jacobi preconditioning and with $\triangle t / \triangle x^{2}=10$. As seen in one dimension, use of the optimal w sequence without multigrid provides only a small improvement over the constant $\omega=1.0$ case. When relaxation with the optimal $\omega$ sequence is combined with multigrid, the solution converges at a spectral radius of 0.89 . This rate is faster than that predicted by the analysis and represents a 14 fold reduction in work as compared to an explicit method.

\section{Concluding Remarks}

Block Jaccbi and block Gauss-Seidel relaxation methods have been analyzed for the solution of the discontinuous Galerkin method applied to diffusion. A Fourier eigenvalue analysis indicates that both preconditioners bound the eigenvalues. In one dimension, the eigenvalues are grouped into well defined regions. both preconditioners place one eigenvalue near zoro, block Jacobi has one eigenvalue near -2 , and all of the remaining eigenvalues are mapped exactly into -1.0 . In two dimension, several eigenvalues are mapped into -1.0 ; however, the remaining eigenvalues are distributed within the bounding region. In general, the eigenvalues become more tightly grouped and the minimum eigenvalue moves closer to zero as $p$ increases. Block Jacobi reduces the ratio of the maximum eigenvalue to the minimum eigenvalue by about an order of magnitude; block Gauss-Seidel reduces the ratio by an additional half an order. Optimal relaxation methods that employ a sequence of under- and over-relaxation are formulated that provided bounded convergence rates of the high frequencies as the time step increases towards infinity. Numerical examples with block Jacobi and multigrid demonstrated convergence rates near the predicted values. When the use of large time steps is appropriate, or when steady state solutions are desired, the methods clescribed here offer more than an order of magnitude reduction in the work.

\section{References}

${ }^{1}$ Harold Atkins, Abdelkader Baggag, Can Ozturan, and Javid Keyes, "Parallelization of an Object-Oriented Unstruc- 
tured Aeroacoustic Solver." Ninth SIAM Conference on Parallel Processing for Scientific Computing, March 22-24. 1999.

${ }^{2}$ Harold Atkins, Chi-Wang Shu, "Quadraturt-Free Implementation of Discontinuous (ialerkin Method for Hyperbolic Equations", AlAA Journal Vol. 36, No. 5, 1998, pp. 775-778.

${ }^{3}$ Harold Atkins. (hi-Wang Shu, "Analysis of the Discontinuous Galerkin Method Applied to the Diffusion Operator", AIAA Paper 99-3306, 1999.

${ }^{4} \mathrm{~J}$. Tinsley Oden, Ivo Babuska, and Carlıs Erik Baumann, "A Discontinuous $h p$ Finite Element Method for Diffusion Problems," Journal of Computational Physics, Vol. 146, 1998, pp. $1-29$.

${ }^{5}$ Bernardo Cockburn, Chi-Wang Shu, "The Local Discontinuous Galerkin Method For Time-Dependent ConvertionDiffusion Systems," SIAM Journal of Numerical Analysis, Vol. 35, 1998, pp. 2440-2463.

${ }^{6}$ Harold Atkins, David Lockard "A High-Order method using Unstructured Grids for the Aeroacoustic Analysis of Realistic Aircraft Configurations", AIA A Paper 99-1945, 1999.

${ }^{7}$ Brandt, A., "Multi-level adaptive solutions to boundaryvalue problems." Mathematics of Computation, Vol 31 . pp. 333$390,1977$.

Table 1 w curve fit parameters and asymptotic convergence rate for block Gauss-Seidel

\begin{tabular}{|c|c|c|c|c|}
\hline$p$ & $\omega_{m}$ & $a$ & $b$ & $\begin{array}{l}\sqrt{G_{g s}\left(\omega_{f}, \sigma(\pi / 2)\right)} \\
\text { for } \lambda=10^{8}\end{array}$ \\
\hline 1 & 2.32 & 1.1858 & 0.314 & 0.427 \\
\hline 2 & 3.85 & 1.1486 & 0.623 & $0.55 \mathrm{l}$ \\
\hline 3 & 6.15 & 1.1144 & 0.777 & $0.5: 31$ \\
\hline 4 & 9.13 & 1.0878 & 0.919 & 0.549 \\
\hline 5 & 12.79 & 1.0781 & 0.917 & 0.557 \\
\hline
\end{tabular}

Table $2 \omega_{f}$ curve fit parameters and asymptotic convergence rate for block Jacobi

\begin{tabular}{|c|c|c|c|c|}
\hline$p$ & $\omega_{m}$ & $a$ & $b$ & $\begin{array}{c}\sqrt{C_{g s}\left(\omega_{f}, \sigma(\pi / 2)\right)} \\
\text { for } \lambda=10^{8}\end{array}$ \\
\hline 1 & 2.77 & 1.1919 & 0.218 & 0.684 \\
2 & 5.26 & 1.1379 & 0.451 & 0.740 \\
3 & 8.76 & 1.1194 & 0.556 & 0.762 \\
4 & 13.25 & 1.0699 & 0.495 & 0.773 \\
5 & 18.75 & 1.0200 & 0.602 & 0.779 \\
\hline
\end{tabular}

Table $3 \sigma^{*}$ and optimal $\omega$ for block Jacobi preconditioning with $p=4$

\begin{tabular}{|c|c|c|c|c|}
\hline$\lambda$ & $\sigma^{*}$ & $\omega_{1}$ & $\omega_{2}$ & $\omega_{3}$ \\
\hline $10^{-2}$ & -0.297 & 0.5302 & 0.87057 & 2.4309 \\
0.1 & -0.0845 & 0.5343 & 0.95946 & 4.6989 \\
1.0 & -0.0308 & 0.5353 & 0.98483 & 6.1458 \\
10.0 & -0.0237 & 0.5354 & 0.98830 & 6.4078 \\
$10^{8}$ & -0.0228 & 0.5355 & 0.98873 & 6.4413 \\
\hline
\end{tabular}

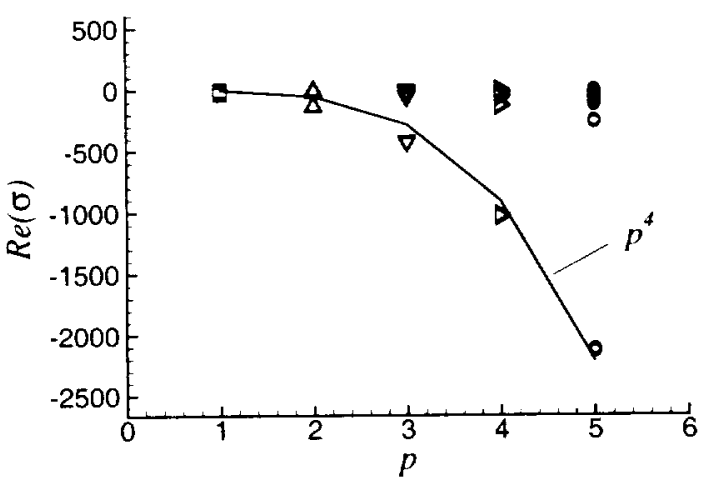

Fig. 1 Eigenvalues of residual operator, $\lambda=1.0$
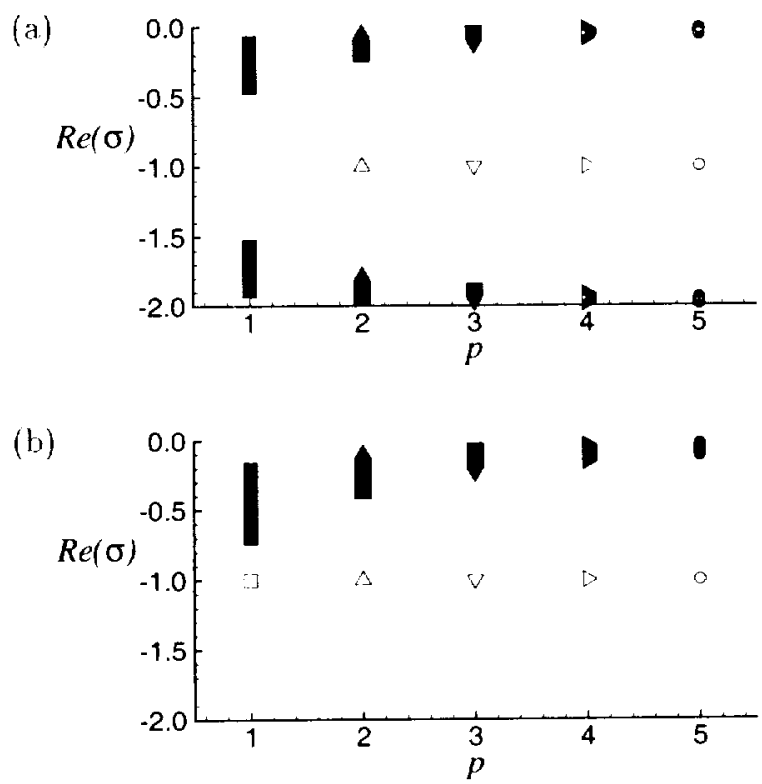

Fig. 2 Eigenvalues of preconditioned residual operator with $\lambda=1.0$ and using a) block Jacobi preconditioning and b) block Gauss-Seidel preconditioning. 
(a)

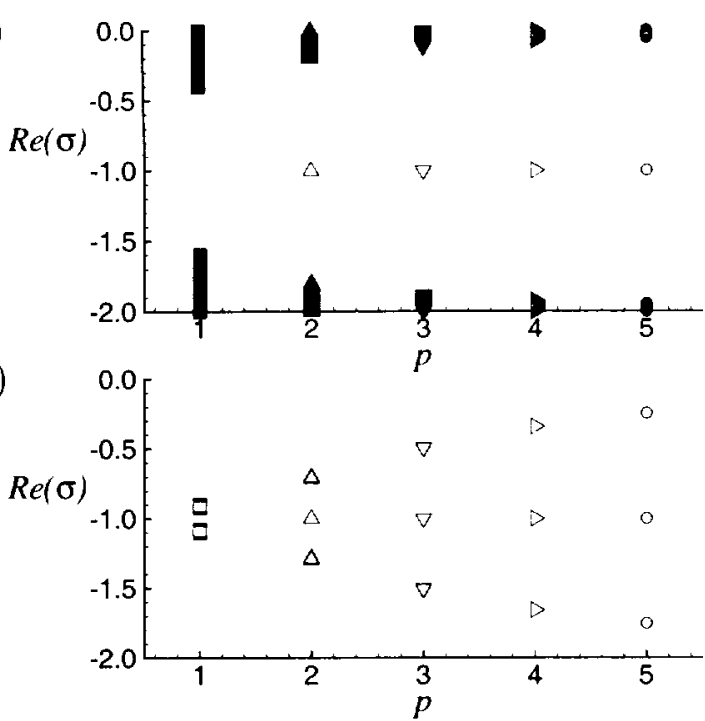

Fig. 3 Eigenvalues of preconditioned residual operator with block Jacobi preconditioning and with: a) $\lambda=100.0$ and b) $\lambda=0.01$.

(a)

(b)
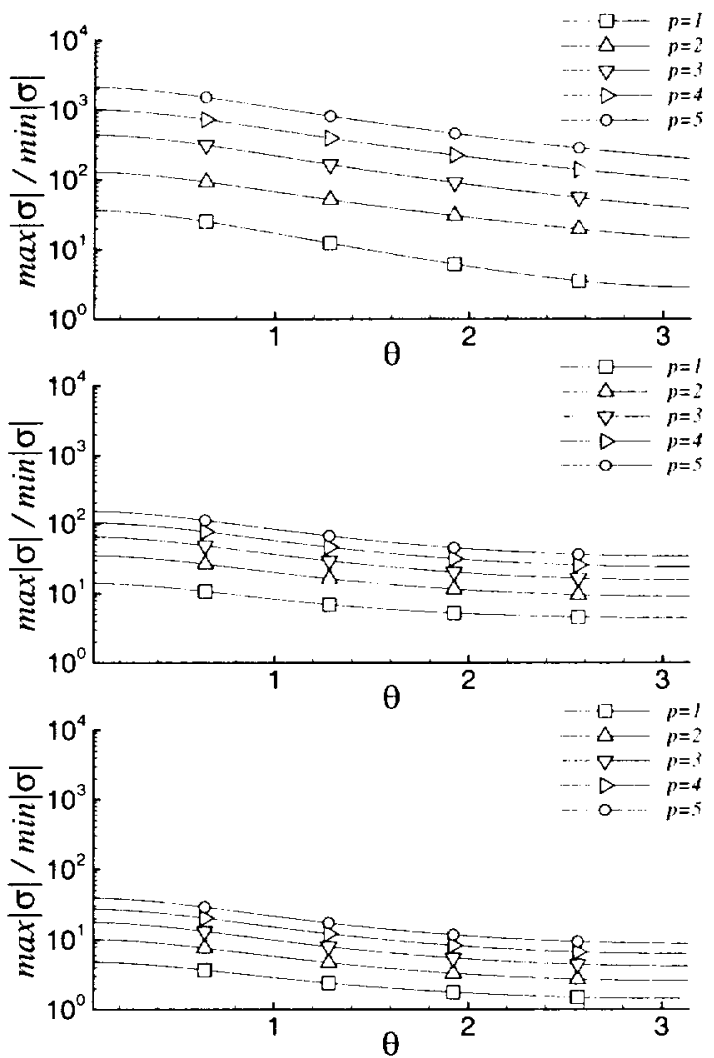

Fig. 4 Ratio of maximum and minimum eigenvalues with $\lambda=1.0$ and a) no preconditioning b) block Jacobi preconditioning and c) block Gauss-Seidel preconditioning.

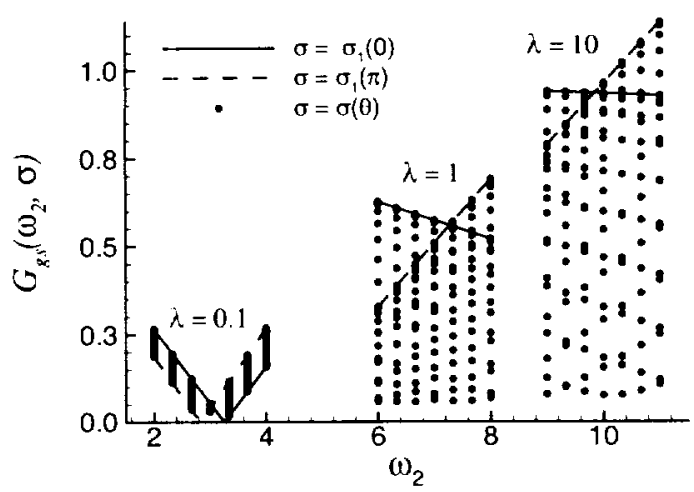

Fig. 5 Optimal $\omega_{2}$ over all $\theta$ located at intersection of $G_{g s}\left(\omega_{2}, \sigma(0)\right)$ and $G_{g_{s}}\left(\omega_{2}, \sigma(\pi)\right)$.

(a)

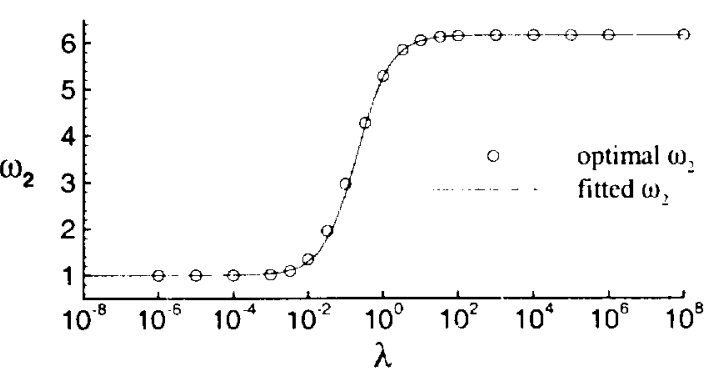

(b)

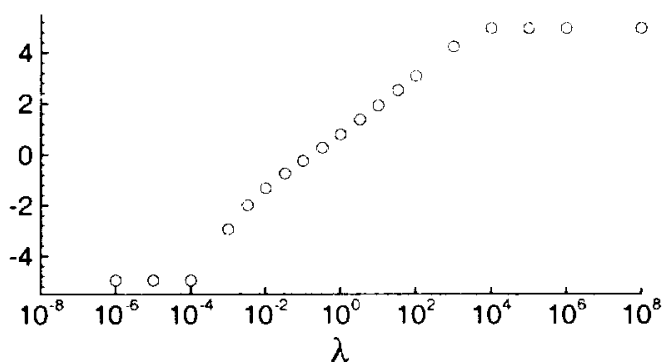

Fig. 6 a) Optimal $\omega$ for all $\theta$ and curve fit, b) linear region for curve fit seen in $\tanh ^{-1}\left(2 * \omega_{f}-\right.$ $\left.\left.\omega_{m}-1\right) /\left(\omega_{m}-1\right)\right)$.

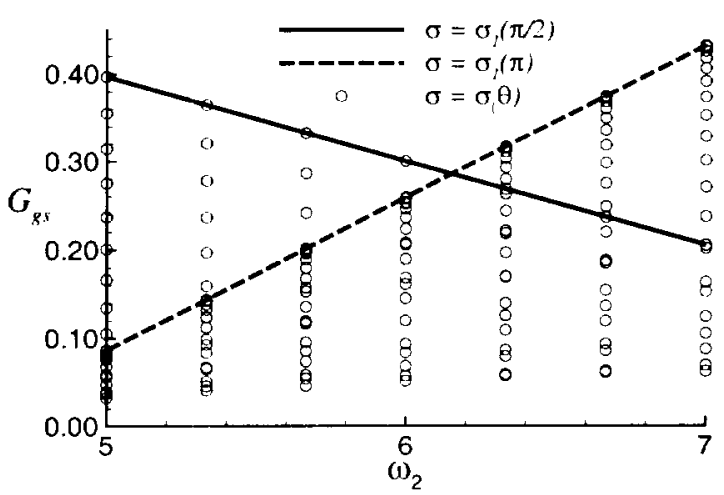

Fig. 7 Optimal $\omega_{2}$ for all $\theta \geq \pi / 2$ located at intersection of $G_{g s}\left(\omega_{2}, \sigma(\pi / 2)\right)$ and ${ }_{i g}\left(\omega_{2}, \sigma(\pi)\right)$. 


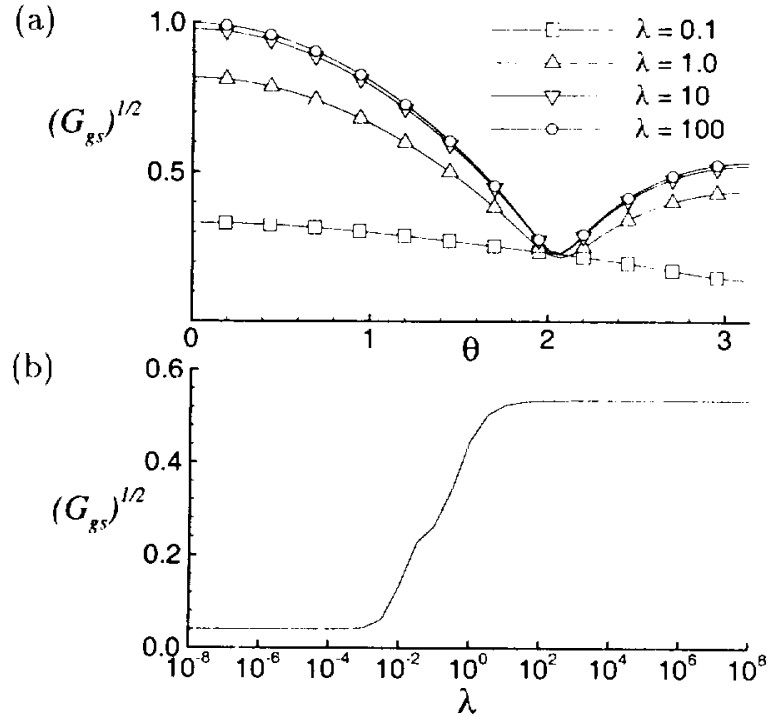

Fig. 8 Anuplification of relaxation with block Gauss-Seidel preconditioning using optimal $\omega$ sequence: a) $\left(G_{g s}\right)^{\frac{1}{2}}$ VS $\theta$ for $\lambda=0.1,1.0,10$ and 100 , b) $\left(G_{g s}\right)^{\frac{1}{2}}$ at $\theta \geq \pi / 2$.

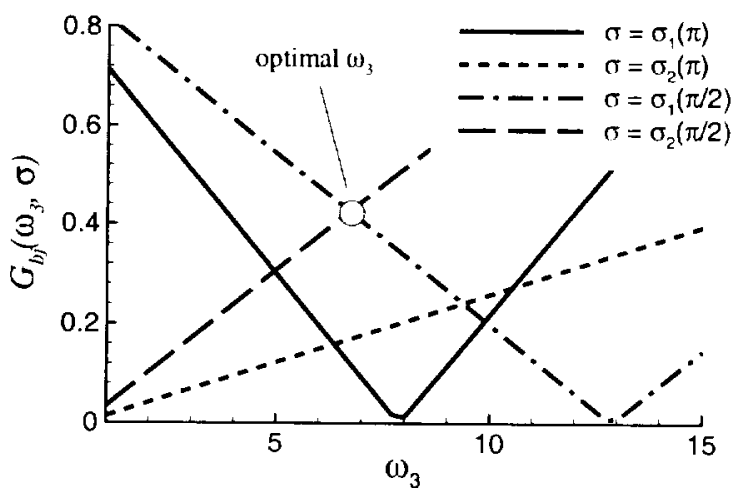

Fig. 9 Optimal $\omega_{2}$ located at the maximum intersection.

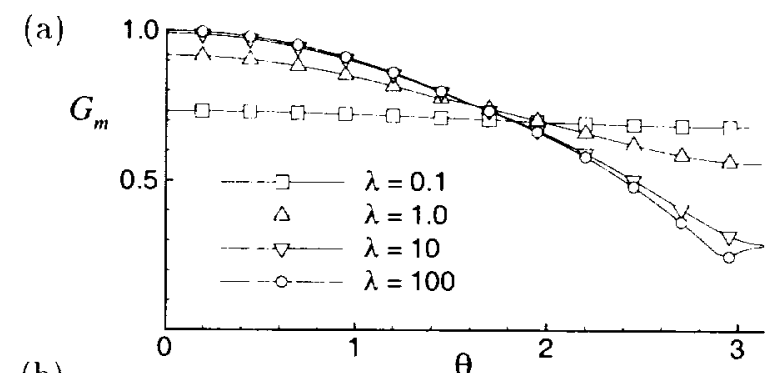

(b)

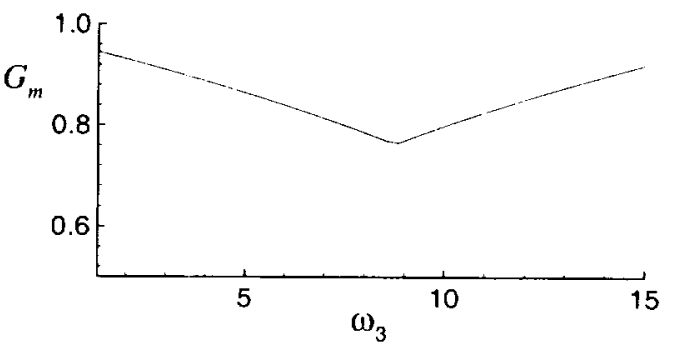

Fig. 10 Amplification of relaxation with block Jacobi preconditioning using optimal $\downarrow$ sequence, $G_{m}=\left(G_{b_{3}}\right)^{1 / 3}:$ a) $G_{m}$ Vs $\theta$ for $\lambda=0.1$. 1.0, 10 and 100 , b) $G_{m}$ insensitive to variation in $\omega_{3}$

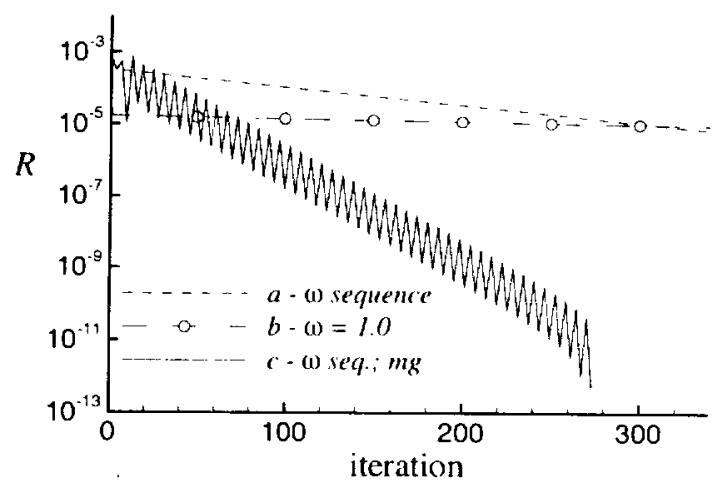

Fig. 11 Convergence of block Jacobi for heat equation in one dimension: a) constant $\omega=1.0$ withont multigrid, b) optimal is sequence without multigrid, c) optimal $\omega$ sequence with multigrid. 

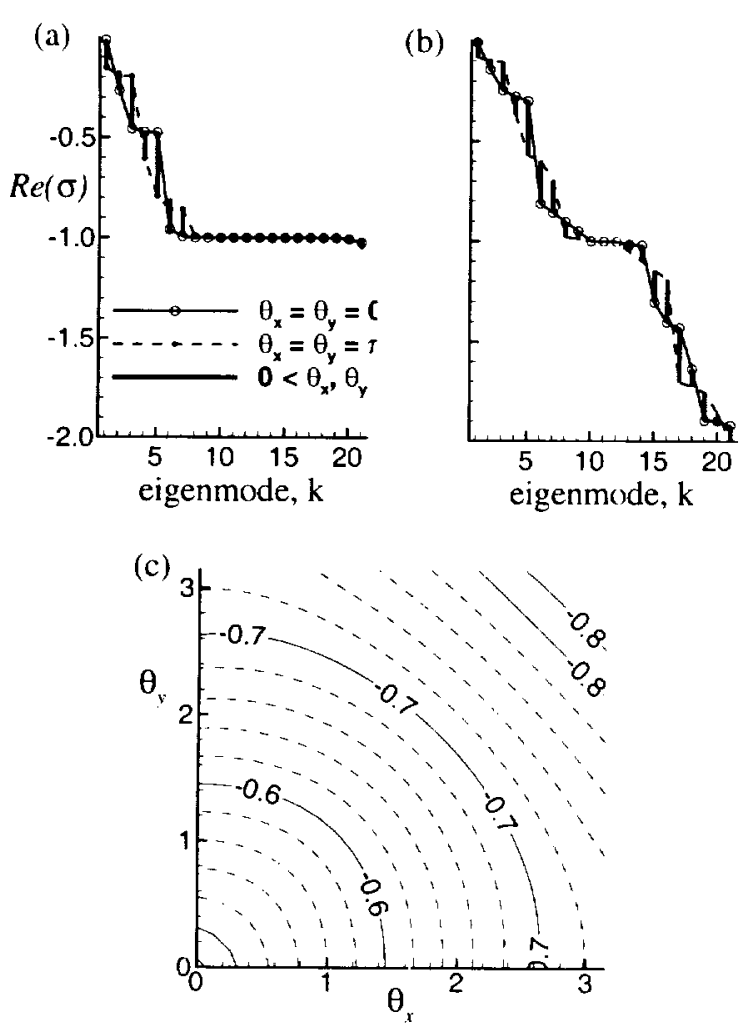

Fig. 12 Eigenvalues of block Gauss-Seidel and block Jacobi in two dimensions: a) distribution of all eigenmodes for block Gauss-Seidel preconditioning, b) distribution of all eigenmodes for block Jacobi preconditioning, c) Fourier distribution of $k=5$ eigenmode for block Gauss-Seidel.

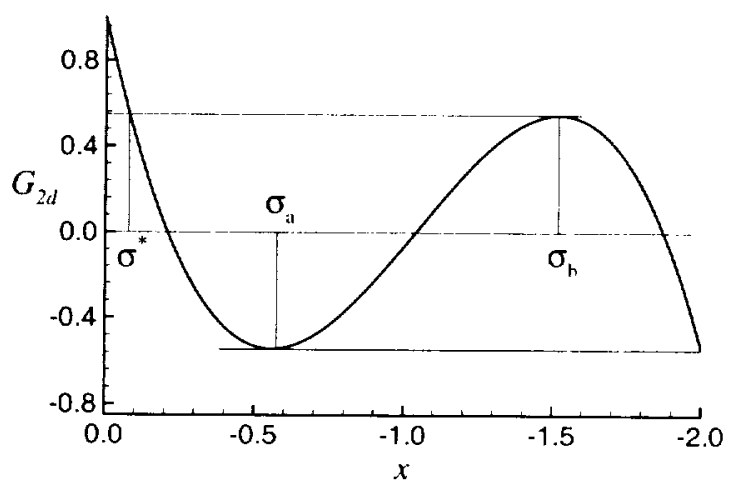

Fig. 13 Optimal amplification for all $\sigma$ such that $\sigma^{*}<\sigma<2.0$.

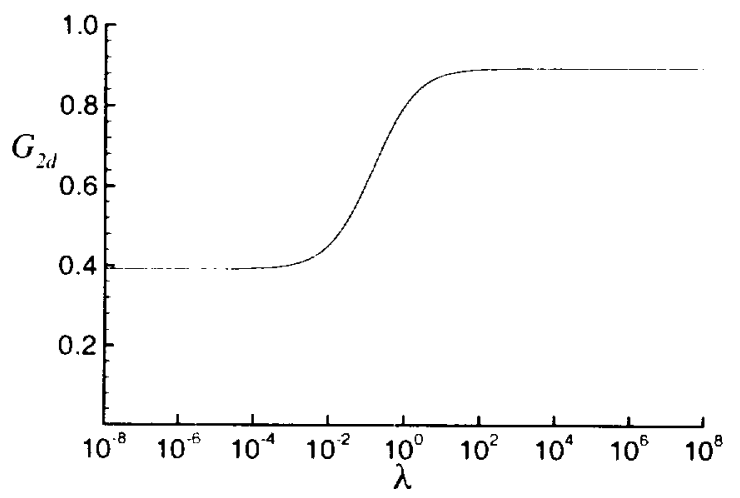

Fig. 14 Predicted asymptotic convergence rate for block Jacobi in two dimensions using the optimal $\omega$ sequence with multigrid, $p=4$. 


\section{NASA STI Acquisitions DAA Authorization}

The following papers (copies enclosed) have been DAA approved as Unclassified, Publicly Available documents:

\section{Meeting Presentations:}

11 th ARM Science Team Meeting Proceedings, 3/19-23/2001, Atlanta, GA:

M.M. Khaiyer, et al.: A 3-Year Climatology of Cloud and Radiative Properties Derived...

A.D. Rapp, et al:: Comparison of Shortwave Cloud Radiative Forcing Derived From...

M.L. Nordeen, et al.: GMS-5 Satellite-Derived Cloud Properties Over the Tropical...

V. Chakrapani, et al.: Improvements in AVHRR Daytime Cloud Detection Over the...

15th AIAA Computational Fluid Dynamics Conf., 6/11-14/2001, Anaheim, CA:

B. Diskin, et al:: Distributed Relaxation for Conservation Discretizations.

H.L. Atkins, et al.: Analysis of Preconditioning and Relaxation Operators for the...

19th AIAA Applied Aerodynamics Conference, 6/11-14/2001, Anaheim, CA:

S.S. Graves, et al.: Dynamic Deformation Measurements of an Aeroelastic Semispan...

37th AIAA/ASME/ASE/ASEE Joint Proplus. Conf. \& Exhib., 7/9-11/2001, Salt Lake City, UT:

R.C. Rogers, et al: Scramjet Tests in Shock Tunnel at Flight Mach 7, 10 and 15 Conditions.

International Symp. on Shock Waves, 23rd, 7/22-27/2001, Ft. Worth, TX:

R.O. Foelsche, et al.: Hypervelocity Capability of the HYPULSE Shock-Expansion...

AIAA Guidance, Navigation, \& Control Conf. \& Exhibit, 8/6-9/2001, Montreal, Canada:

S. Mondoloni, et al.: An Airborne Conflict Resolution Approach Using A Genetic...

\section{Journal Article:}

J.W. Wilson, et al.: Approach and Issue Relating to Shield Material...(Mat'ls \& Desgn, 2001).

X. Dong, et al.: Arctic Stratus Cloud Properties and Their Effect on the...(JOG 2001).

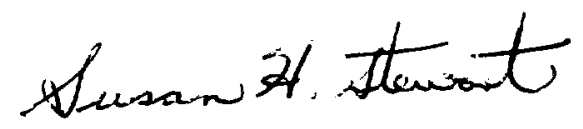

Susan H. Stewart

DAA Representative

NASA Langley Research Center

s.h.stewart@larc.nasa.gov

Mail Stop 196

phone: (757) 864-2518

Hampton, VA 23681-2199 